\title{
The Risk of Carotid Plaque Instability in Patients with Metabolic Syndrome: It is Higher in Women with Hypertriglyceridemia
}

\section{Francesca Servadei}

University of Rome Tor Vergata: Universita degli Studi di Roma Tor Vergata

\section{Lucia Anemona}

University of Rome Tor Vergata: Universita degli Studi di Roma Tor Vergata

Marina Cardellini

University of Rome Tor Vergata: Universita degli Studi di Roma Tor Vergata

\section{Manuel Scimeca}

University of Rome Tor Vergata: Universita degli Studi di Roma Tor Vergata

Manuela Montanaro ( $\nabla$ manuela.montanaro@uniroma2.it )

University of Rome Tor Vergata: Universita degli Studi di Roma Tor Vergata 0001-8441-3731

\section{Valentina Rovella}

University of Rome Tor Vergata: Universita degli Studi di Roma Tor Vergata

\section{Francesca Di Daniele}

University of Rome Tor Vergata: Universita degli Studi di Roma Tor Vergata

\section{Erica Giacobbi}

University of Rome Tor Vergata: Universita degli Studi di Roma Tor Vergata Jacopo Maria Legramante

University of Rome Tor Vergata: Universita degli Studi di Roma Tor Vergata Annalisa Noce

University of Rome Tor Vergata: Universita degli Studi di Roma Tor Vergata

Rita Bonfiglio

University of Rome Tor Vergata: Universita degli Studi di Roma Tor Vergata

\section{Patrizia Borboni}

University of Rome Tor Vergata: Universita degli Studi di Roma Tor Vergata

\section{Nicola Di Daniele}

University of Rome Tor Vergata: Universita degli Studi di Roma Tor Vergata

\section{Arnaldo Ippoliti}

University of Rome Tor Vergata: Universita degli Studi di Roma Tor Vergata

\section{Massimo Federici}

University of Rome Tor Vergata: Universita degli Studi di Roma Tor Vergata 


\section{Alessandro Mauriello}

University of Rome Tor Vergata: Universita degli Studi di Roma Tor Vergata https://orcid.org/00000002-7351-5676

\section{Original investigation}

Keywords: Metabolic syndrome, carotid, histology, hypertriglyceridemia, post-menopause

Posted Date: February 2nd, 2021

DOl: https://doi.org/10.21203/rs.3.rs-165616/v1

License: (c) (i) This work is licensed under a Creative Commons Attribution 4.0 International License. Read Full License 
1 The risk of carotid plaque instability in patients with metabolic syndrome:

\section{2 it is higher in women with hypertriglyceridemia}

3 Francesca Servadei ${ }^{1, \#}$, Lucia Anemona ${ }^{1, \#}$, Marina Cardellini ${ }^{2, \#}$, Manuel Scimeca ${ }^{1,3,4}$, Manuela

4 Montanaro $^{1}$, Valentina Rovella ${ }^{5}$, Francesca Di Daniele ${ }^{6}$, Erica Giacobbi ${ }^{1}$, Iacopo Maria

5 Legramante $^{2}$, Annalisa Noce ${ }^{2}$, Rita Bonfiglio ${ }^{1,7}$, Patrizia Borboni ${ }^{2}$, Nicola Di Daniele ${ }^{2}$,

6 Arnaldo Ippoliti ${ }^{8}$, Massimo Federici $^{2}$ and Alessandro Mauriello ${ }^{1,9}$

$7 \quad$ \#Francesca Servadei, Lucia Anemona and Marina Cardellini equally first author.

$9{ }^{1}$ Anatomic Pathology, Department of Experimental Medicine, University of Rome Tor 10 Vergata, Via Montpellier 1, 00133 Rome, Italy;

$11{ }^{2}$ Department of Systems Medicine, University of Rome Tor Vergata, Italy

$12{ }^{3}$ San Raffaele University, Via di Val Cannuta 247, 00166 Rome, Italy;

${ }^{4}$ Saint Camillus International University of Health Sciences, Via di Sant'Alessandro, 8, 00131

14 Rome, Italy;

15

${ }^{5}$ UOC of Internal Medicine - Center of Hypertension, University of Rome Tor Vergata, Italy

${ }^{6} \mathrm{PhD}$ School of Applied Medical, Surgical Sciences, University of Rome Tor Vergata, Italy

$17{ }^{7}$ Fondazione Umberto Veronesi (FUV), Piazza Velasca 5, 20122 Milano (MI), Italy;

${ }^{8}$ Vascular Surgery, Department of Biomedicine and Prevention, University of Rome Tor 19 Vergata, Italy

\footnotetext{
${ }^{9}$ Tor Vergata Oncoscience Research (TOR), University of Rome "Tor Vergata", Italy.
} 


\section{Corresponding Author}

24 Prof. Alessandro Mauriello, MD

Department of Experimental Medicine, University "Tor Vergata", Via Montpellier 1, Rome (RM) 00133 Italy; Email: alessandro.mauriello@uniroma2.it phone: +39 0620903908

\section{Abstract}

Background: Metabolic syndrome certainly favors growth of carotid plaque; however, it is uncertain if it determines plaque destabilization. Furthermore, it is likely that only some components of metabolic syndrome are associated with increased risk of plaque destabilization. Therefore, we evaluated the effect of different elements of metabolic syndrome, individually and in association, on carotid plaques destabilization.

Methods: A total of 186 carotid endarterectomies from symptomatic and asymptomatic patients were histologically analysed and correlated with major cardiovascular risk factors.

36 Results: Metabolic syndrome, regardless of the cluster of its components, is not associated with a significant increase in risk of plaque destabilization, rather with the presence of stable plaques. The incidence of unstable plaques in patients with metabolic syndrome is quite low (43.9\%), when compared with that seen in the presence of some risk factors, but significantly increases in the subgroup of female patients with hypertriglyceridemia, showing an odds ratio of $3.01(\mathrm{CI} 95 \% 0.25-36.30)$.

42 Conclusions: Our data may help to identify patients with real increased risk of acute cerebrovascular diseases and may support the hypothesis that the control of 
hypertriglyceridemia should be a key point on prevention of carotid atherosclerotic plaque destabilization, especially in post-menopausal female patients.

46

47 Keywords: Metabolic syndrome, carotid, histology, hypertriglyceridemia, post-menopause.

\section{Background}

50

Many studies have demonstrated that Metabolic Syndrome (MetS), defined as a cluster of interconnected metabolic risk factors (including abdominal obesity, elevated fasting glucose, hypertriglyceridemia, hypertension, and low High-Density Lipoprotein (HDL) cholesterol levels), increases the risk for atherosclerosis and cardiovascular disease [1-6]. In addition, the recent interest of the scientific community has focused on the possible involvement of insulin resistance as a linking factor [7-10].

Atherosclerosis is a chronic inflammatory disease, to whose clinical manifestations multiple cellular and systemic events contribute [11]. Two types of atherosclerotic disease are described: a stable form (with low embolic risk, constant and slow growth of the plaque over time) and an unstable form (with a high embolic risk, linked to the presence of a significant inflammation which causes the rupture of the plaque, with thrombosis and subsequent fragment's detachment resulting in embolic complications and leading to acute clinical manifestations) [12-14]. Despite it is known that the cardiovascular risk factors certainly favor the development and growth of atherosclerotic plaque, it is not yet fully understood exactly which in particular favor its destabilization by accelerating plaque growth with consequent rupture and thrombosis [1517]. It is possible to hypothesize that the main cardiovascular risk factors can play a crucial role in the destabilization process of atheromatous lesions through the modification of the histological composition of fibroatheromatous plaques. The identification of specific risk 
factors associated with plaque destabilization is particularly important in the prevention and treatment of cerebrovascular syndromes. According to American Heart Association (AHA) and American College of Cardiology (ACC) guidelines, severe carotid stenosis is the main predisposing condition for acute cerebrovascular events and it still represents the most important criterion to identify patients who need surgical treatment [18-21]. Nevertheless, whether it is necessary to perform carotid endarterectomy in all asymptomatic patients with stenosis $>70 \%$ (even if most of them have stable plaque that is not at risk of thromboembolism), is still an open question. Indeed, it is now clear that the degree of stenosis alone is not sufficient to accurately identify patients at high risk of developing an acute cerebrovascular event. Thus, in this scenario, the identification of risk factors correlated with plaque destabilization becomes necessary to calculate the most real probability of ischemic cerebrovascular complications, stratifying patients with carotid atherosclerosis.

Previous clinical studies have consistently demonstrated a significant association between MetS and incidence of ischemic stroke [2, 6, 22]. However, the relationship between MetS and the morphological characteristics of carotid plaque instability remains uncertain. Indeed, previous studies have mainly used the imaging, in particular the measurement of carotid Intimal-Medial Thickness (IMT) to assess the risk of ischemic stroke [8, 23-24]. This represents a valid approach to evaluate the degree of atherosclerosis, but it does not provide information on the possible destabilization of plaques, that can only be assessed by histological methods. Furthermore, it has not yet been clarified which of plaque's individual components were associated with an increased stroke risk. Therefore, in this study we investigate each main factor involved in MetS, in order to estimate their impact on the destabilization of the carotid plaque. Specifically, to quantify their possible synergistic effect, the relative risk of different components of metabolic syndrome, individually and in association with each other, were calculated. 


\section{$\underline{\text { Cases selection and histology }}$}

95

A total of 186 carotid plaques from symptomatic (major stroke or transient ischemic attack TIA) and asymptomatic patients submitted to surgical carotid endarterectomy (CEA) at the University of Tor Vergata (Rome, Italy) from 2016 to date were analysed. The sampling collection and analysis methods have been previously reported [13]. In short, samples were fixed in $10 \%$ buffered formalin, briefly decalcified using the Surgipath Decalcifer II (LEICA, Western Road, Stratford Upon Avon, UK) in order to allow the cutting without artifacts and to preserve the recognition of perfect morphology of the plaque, cut transversely every $5 \mathrm{~mm}$, embedded in paraffin, and stained with haematoxylin-eosin. Only intact carotid plaques, from patients with a complete clinical and laboratory assessment of the major cardiovascular risk factors were histologically analysed and included in the study.

According to the modified AHA classification, atherosclerosis plaques have been histologically distinguished into unstable and stable [25]. Unstable plaques consisted of: (a) thrombotic plaques associated with rupture or erosion of the cap; (b) healed plaque with a thrombus in organization; (c) vulnerable plaque or thin-cap fibro-atheroma (TCFA) characterized by a fibrous cap less than $165 \mu \mathrm{m}$ thick, heavily infiltrated by macrophages CD68 positive ( $>25$ per high magnification field), without plaque rupture. The other plaques, classified as stable, included: (a) fibroatheromata, such as plaques with a large lipidic necrotic core and thick non-inflamed cap, (b) fibrocalcific plaques with large calcification without extensive inflammation and (c) fibrous plaques mainly constituted by fibrous tissue (Figure 1). Immunohistochemical analysis was performed in all plaques in order to characterize the inflammatory infiltrate using CD68 antibody anti-monocyte/macrophages cells (rabbit monoclonal, clone KP-1; Ventana, Tucson, AZ, USA) and CD3 antibody anti-T cells (rabbit monoclonal, clone 2GV6; Ventana). 
118 Histopathologic examination was performed, utilizing the definitions reported above, by two

119 blinded pathologists (A.M., F.S.). Inter-observer reliability was $>98 \%$.

120

121 Clinical records were reviewed for all cases in order to determine risk factors profile.

122 According to AHA Scientific Statements [1] the presence of any 3 of 5 following conditions

123 constitutes a diagnosis of metabolic syndrome: (a) elevated waist circumference (abdominal obesity) $>94 \mathrm{~cm}$ in men and $>80 \mathrm{~cm}$ in women; (b) hypertriglyceridemia: patients with serum triglycerides levels $\geq 150 \mathrm{mg} / \mathrm{dL}$ ( $>1.70 \mathrm{mmol} / \mathrm{L}$ ); (c) reduced HDL-C, $<40 \mathrm{mg} / \mathrm{dL}$ in men or $<50 \mathrm{mg} / \mathrm{dL}$ in women; (d) elevated blood pressure, systolic $\geq 130$ and/or diastolic $\geq 85 \mathrm{~mm}$

$127 \mathrm{Hg}$; (e) elevated fasting glucose $\geq 100 \mathrm{mg} / \mathrm{dL}$.

128 The presence of hypertension was also evaluated according to the American College of 129 Cardiology/American Heart Association (ACC/AHA) [26], the European Society of 130 Cardiology (ESC) and European Society of Hypertension (ESH) Guidelines [27].

131 The other following major risk factors were considered: (a) hypercholesterolemia: patients with 132 total cholesterol level $>200 \mathrm{mg} / \mathrm{dL}(>5.18 \mathrm{mmol} / \mathrm{L})$; (b) diabetes mellitus: patients with fasting 133 blood glucose $>126 \mathrm{mg} / \mathrm{dL}$ and/or following oral treatment or insulin therapy; (c) patients with 134 tobacco dependence were categorized as: smokers, if the consumption was more than 10 135 cigarettes/day, while who had stopped smoking for $>5$ years was considered as non-smokers.

\section{Statistical analysis}

137 Data were analyzed using SPSS version 16.0 (SPSS Inc, Chicago, Ill) software. Continuous 138 variables were expressed as the mean $\pm \mathrm{SD}$ or $\pm \mathrm{SE}$. The Shapiro-Wilk test was used to 139 statistically assess the normal distribution of the data. Comparisons between continuous 140 variables were performed using the independent Student t-test or the Wilcoxon rank sum test. 141 Categorical data were analysed using the chi-square test or the Fisher exact test. 
142 Multivariate analysis using stepwise logistic regression (using the "enter" method for variable

143 selection) was utilized to identify independent risk factors which significantly correlate with

144 the presence of plaque destabilization. The following variables were included: age, gender,

145 hypertension, diabetes, smoking habit, hypercholesterolemia, low HDL and

146 hypertriglyceridemia, abdominal obesity, metabolic syndrome. Multivariate analysis was

147 performed in 3 models: (1) using the definition of hypertension according the ACC/AHA, (2)

148 according the pressure cut-off of ESC/ESH Guidelines; (3) when the metabolic syndrome was

149 considered in the multivariate analysis, hypertension, diabetes, hypertriglyceridemia, low

150 HDL, and abdominal obesity were excluded. The odds ratio of an unstable plaque for the

151 different risk factors was evaluated by logistic regression using the value of EXP (B), where B

152 represents the logistic coefficient.

153 Moreover, multivariate logistic regression was also used in order to evaluate the effect of each

154 component of metabolic syndrome in plaque destabilization. In this analysis only patients with

155 the metabolic syndrome were considered.

156 A 2-tailed $\mathrm{p}$ value $<0.05$ was considered statistically significant.

157 Results

158 Baseline data

159 Baseline data of patients are reported in Table 1.(Please, insert Table 1 here).

160 The mean age of 186 patients at time of surgical CEA was $72.6 \pm 8.6$ years, $131(70.4 \%)$ were

161 male and $55(29.6 \%)$ were female. Seventy-two (38.7\%) patients were symptomatic (affected

162 by ipsilateral major stroke or TIA), while 114 (61.3\%) were asymptomatic who underwent

163 CEA for high grade carotid stenosis $(>60 \%)$, assessed by echography or, in rare cases, by

164 bilateral CT angiography.

165 A metabolic syndrome was observed in 85 out of 186 patients (45.7\%). All patients included

166 in this study presented at least one risk factor. Among single risk factors, the hypertension was 
the most frequently observed when it was evaluated according to both the ACC/AHA (162 patients, $87.1 \%$ ) and to the ESC/ESH criteria (112 patients, $60.2 \%)$. The low incidence of hypercholesterolemia (only $15.6 \%$ of cases) may probably be explained by the 128 patients

$170(68.8 \%)$ who underwent statins treatments. Continuous treatment with aspirin $(100 \mathrm{md} / \mathrm{die})$

171 was administered to all patients in the post-operative and follow-up periods.

172 In 82 out of 186 patients (44.1\%) unstable plaques were found (Figure 1). They consisted in 17348 thrombotic plaques (all from symptomatic patients) associated with rupture of a thin fibrous 174 cap rich in inflammatory cells, 16 TCFA (10 from symptomatic and 6 from asymptomatic 175 patients) and 16 plaques with an organized acute thrombus (all symptomatic) characterized by 176 a network of large, thin-walled vascular channels and a variable number of macrophagic cells 177 loaded with hemosiderin within the area of an acute thrombus. In the remaining 2 unstable 178 plaques, a calcified nodule was found protruding into the lumen covered by extremely thin 179 fibrous cap. The remaining 104 carotids (55.9\% of cases) showed a stable plaque, characterized 180 by a variable lipid-necrotic core containing extracellular lipid, cholesterol crystals and necrotic 181 debris covered by a thick fibrous cap with few inflammatory cells (Figure 1). In 33 plaques 182 (both stable and unstable) a large calcification was observed.

\section{$183 \quad$ Plaque instability and risk factors}

184 The presence of unstable carotid plaques was not correlated with the finding of specific risk 185 factors, as demonstrated by the uni- and multivariate analysis (see Table 2).

186 Table 2. Plaque instability and risk factors

$\begin{array}{ccccc}\text { Stable } & \text { Unstable } & \text { Odds ratio } & \mathrm{P} & \mathrm{P} \\ \text { plaques } & \text { plaques } & (95 \% \mathrm{CI}) & \text { uni- } & \text { multi- } \\ (104 \text { cases }) & (82 \text { cases }) & & \text { variate } & \text { variate } \\ & & & \text { analysis } & \text { analysis }\end{array}$


$\begin{array}{llllll}\text { Age }(\mathrm{yrs} \pm \mathrm{SD}) & 72.5 \pm 8.1 & 72.8 \pm 9.3 & 0.99(0.96-1.03) & 0.80 & 0.79\end{array}$

Gender

$\begin{array}{llllll}\text { Male } & 65(62.5 \%) & 66(80.5 \%) & 0.41(0.20-0.82) & 0.008 & 0.01\end{array}$

Female $\quad 39(37.5 \%) \quad 16(19.5 \%)$

$\begin{array}{llllll}\text { Hypertension acc. AHA } & 90(86.5 \%) & 72(87.8 \%) & 1.38(0.54-3.56) & 0.80 & 0.50\end{array}$

$\begin{array}{llllll}\text { Diabetes } & 48(46.2 \%) & 32(39.0 \%) & 0.69(0.35-1.36) & 0.33 & 0.28\end{array}$

$\begin{array}{llllll}\text { Smoking habit } & 23(22.1 \%) & 17(20.7 \%) & 0.81(0.37-1.75) & 0.82 & 0.58\end{array}$

$\begin{array}{llllll}\text { Hypercholesterolemia } & 17(16.3 \%) & 12(14.6 \%) & 0.72(0.29-1.76) & 0.75 & 0.47\end{array}$

$\begin{array}{llllll}\text { Hypertriglyceridemia } & 35(33.7 \%) & 35(42.7 \%) & 1.75(0.87-3.51) & 0.21 & 0.12\end{array}$

$\begin{array}{llllll}\text { Low-HDL } & 48(46.2 \%) & 38(46.3 \%) & 1.22(0.62-2.40) & 0.98 & 0.57\end{array}$

$\begin{array}{llllll}\text { Abdominal obesity } \quad 20(19.2 \%) & 9(11.0 \%) & 0.46(0.19-1-12) & 0.12 & 0.09\end{array}$

$\begin{array}{llllll}\text { Hypertension acc. ESC } \quad 62(59.6 \%) & 50(61.0 \%) & 1.11(0.58-2.14) & 0.85 & 0.74\end{array}$

$\begin{array}{llllll}\text { Metabolic syndrome } & 49(47.1 \%) & 36(43.9 \%) & 0.86(0.46-1.58) & 0.66 & 0.62\end{array}$

187

188 Only a significant correlation with the gender was observed, as males showed a higher 189 incidence of unstable plaques than females $(\mathrm{p}=0.01)$. In fact, unstable plaques were observed 190 in 66 of the $131(50.45 \%)$ male patients undergoing CEA and only in 16 of the $55(29.1 \%)$ 191 female patients.

192 In particular, the incidence of unstable plaques in patients with metabolic syndrome was $42.4 \%$

193 (36 out of 85 cases), with no significant differences compared to that of stable plaques ( $p=$ $1940.62)$.

195 When only the individual risk factors were considered in the statistical analysis, the greatest 196 odds ratio for an unstable plaque was observed in patients with hypertriglyceridemia (1.75, $19795 \%$ CI $0.87-3.51$ ) or hypertension (according to ACC/AHA) (1.38, 95\% CI 0.54-3.56). 
198 Considering the metabolic syndrome instead of hypertension, diabetes, hypertriglyceridemia, 199 low HDL-C and abdominal obesity, a low odds ratio for plaque instability $(0.86,95 \%$ CI 0.46 200 1.58) were found by multivariate analysis.

\section{Components of metabolic syndrome and plaque instability}

202 Taking into account only the patients with metabolic syndrome, we evaluated the effect of 203 single risk factors on plaque destabilization.

204 Patients with hypertriglyceridemia were those who presented a greater risk of destabilization of plaques (an odds-ratio of 1.43 , CI95\% 0.44 - 3.52). Since a significant inverse correlation between age and triglyceridemia values was observed $(r=-0.44, p=0.001)$, the risk was calculated in two subgroups of patients with metabolic syndrome, with an age of \pm 70 years old. Patients $<70$ years old had an odds ratio of 1.55 (CI95\% $0.25-9.54)$, while in the elderly it was reduced to $1.00(\mathrm{CI} 95 \% 0.17-5.76)$. The risk was significantly increased in women with hypertriglyceridemia compared to males. In fact, the odds ratio was 1.28 (CI95\% 0.37 - 4.48) in the male subgroup and 3.01 (CI95\% 0.25 - 36.30) in the female one.

212 The other possible combinations of the metabolic syndrome components did not achieve a 213 significant increase in risk.

\section{Discussion}

215 The results obtained in our study show that MetS is not associated with a significant increase 216 in the risk of carotid plaque destabilization, regardless of the analyzed risk factors. On the

217 contrary, MetS showed a correlation with the presence of stable plaques according to the 218 histological features released by the ACC/AHA [26]. Indeed, the incidence of unstable plaques in patients with MetS is rather low (43.9\%), when compared with that observed in the presence 220 of other individual risk factors such as hypertension. However, the risk of carotid plaque 
221 destabilization significantly increases in the subgroup of female patients with

222 hypertriglyceridemia, showing an odds ratio of 3.01 .

223 Carotid stenosis still remains the criterion of choice to undergo a preventive endarterectomy, 224 especially in asymptomatic patients [18-21]. According to our previous studies, results of the 225 present study showed that more than $50 \%$ of asymptomatic patients undergoing surgery had a 226 stable carotid plaque that evolves very slowly over time [13-14, 28]. This problem is 227 particularly important in those asymptomatic patients with unilateral carotid stenosis who have no other comorbidities that may increase the risk of an ischemic stroke. The prevention of atherosclerosis is based on the control of the major cardiovascular risk factors and each patient has many of them, whose interaction must be taken into consideration. The various risk factors probably promote the slow growth of the carotid plaque. However, there are conflicting data which lead to the hypothesis that they can significantly determine not only the plaque growth but also its rupture and thrombosis.

The results of the multivariate analysis reported in our study showed that none of the considered risk factors were independently associated with an increased risk of destabilization of the carotid plaque (Table 2). In particular, unstable carotid plaques were more frequent in hypertensive patients but, according to both the AHA [26] and ESC Guidelines [27], the assessed incidence of hypertension was equally high even in patients with stable plaques ( $p=0.50$ and 0.74 , respectively). About $40 \%$ of patients with unstable plaque were also affected by the MetS itself; nevertheless, a similar incidence was observed in those with stable plaque

$241(\mathrm{p}=0.62)$. However, our results do not contradict some clinical meta-analyses, that propose the

242 MetS as a relevant risk of developing cerebrovascular disease [5-6, 29]. Among them, Mottillo

243 et al. performed a large clinical meta-analysis by investigating data of 87 studies, comprising 244951.083 subjects [29]. The increased risk, as our results have demonstrated, is not due to MetS 245 itself but it varies on the basis of the combination of MetS components present in each patient. 
246 Only few and contradictory studies have assessed all possible combinations of MetS

247 components in order to determine which elements are most strongly associated with

248 atherosclerosis [8, 30-32]. In particular, Golden et al. found a strong independent association

249 between selected groupings of MetS components and excess carotid intimal-medial thickness,

250 suggesting a synergy for atherosclerosis risk beyond what would have been expected from a

251 merely additive effects [8]. The evaluation of carotid intimal-medial thickness represents only

252 a subclinical assessment of atherosclerosis [8, 23-24] and does not distinguish between a stable

253 plaque and ones with risk of rupture and thrombosis. A precise risk evaluation of plaque rupture

254 can only be performed with histological methods (as in the present study) or with high-

255 definition imaging approaches.

256 The major component of MetS that significantly correlates with an increased risk of 257 destabilization of the carotid plaque was the hypertriglyceridemia. In our series, 258 hypertriglyceridemia represents the major component of dyslipidemia associated with the 259 progression of atherosclerotic carotid plaque (Table 2). In fact, it should be emphasized that in 260 our study, patients underwent endarterectomy (symptomatic or asymptomatic but potentially 261 with risk of acute cerebrovascular syndrome), showed a very low incidence of hypercholesterolemia ( $15.6 \%$ of cases). This can be explained by the fact that 128 out of 186 patients (68.8\%) were taking statins which, as it is well known, have a lowering effect on both LDL-C levels and anti-inflammatory effects.

265 Recent studies have long considered that elevated triglyceride levels increase the risk of 266 cardiovascular disease that persist despite statin treatment [2, 33-34]. Normal or low 267 triglyceride levels are associated with reduced cardiovascular event, as demonstrated by PROVE-IT TIMI (Pravastatin or Atorvastatin Evaluation and Infection Therapy-Thrombolysis In Myocardial Infarction) trial [35]. Patients who received statin treatment after hospitalization

270 for acute coronary syndrome had a lower risk of further coronary heart disease events if 
271 triglyceride levels were $<150 \mathrm{mg} / \mathrm{dL}$ [35]. These results were confirmed by the recent 272 REDUCE-IT (Reduction of Cardiovascular Events with Icosapent Ethyl-Intervention) clinical

273 trial carried out on 8170 patients under statin therapy, with elevated fasting triglyceride levels

274 and normal LDL-C levels [36]. This trial demonstrated a significant reduction in ischemic 275 events in subjects taking Icosapent Ethyl (a molecule which decreases triglyceride levels 276 without modifying LDL-C) as compared to subjects taking placebo, thus demonstrating how 277 the reduction of triglyceride levels is associated with a lower risk of cardiovascular events among subjects with normal LDL-C levels [36]. Despite the normalization of LDL-C levels for the intake of statins, our results confirm that also in carotid district the increase in triglycerides 280 is associated with an increase in the destabilization of atherosclerotic plaque, providing an evident morphological basis.

Hypertriglyceridemia could favor the destabilization of the carotid plaques by increasing local inflammation, damaging the endothelium, and stimulating the expression of cellular adhesion molecules [37-38]. High levels of triglycerides are also associated with increased remnant lipoprotein particles that induce the expression of pro-inflammatory cytokines (TNF- $\alpha$, IL-6, VCAM1, etc.) which have a cytotoxic effect on the endothelium [39]. The observation that the risk of plaque instability is higher in female patients with metabolic syndrome and hypertriglyceridemia, than in those without hypertriglyceridemia, confirms the conclusions of a recent meta-analysis by Li et al. [6]. The study reported that patients with MetS had a significantly higher risk of incident stroke, as compared to those without MetS, and that this effect was most remarkable among females (RR, 1.83 [95\% CI, 1.31-2.56]) compared with males (RR, 1.47 [95\% CI, 1.22-1.78]). Sex-difference in cardiovascular disease is now widely accepted [40]. Most likely, sex hormones play an atherosclerotic protective role in women, due to their action on endothelial function and lipid homeostasis. Estrogens might have plaque stabilization properties and 
296 effects on plaque inflammatory status [41] [42]. The effects of estrogens are probably age-

297 dependent, reducing inflammation in younger age, while an opposite pro-inflammatory effect

298 could be observed in older women, as those analyzed in the present study [43] [44].

\section{Conclusions}

300 Data here reported suggest that a better understanding of how each individual component of

301 MetS influences the stroke risk correlated to carotid plaque destabilization, rupture, and

302 thrombosis, may help to stratified patients according to the real risk of acute cerebrovascular

303 diseases. Moreover, our results support the hypothesis that the control of hypertriglyceridemia

304 should be a key point on prevention of the destabilization of atherosclerotic carotid plaque,

305 especially in post-menopausal female patients. However, data on sex-difference are still

306 limited, and further studies are recommended to better define which differences may have

307 implications for clinical prevention and management of acute complications of carotid artery

308 disease.

\section{List of abbreviations}

310 MetS: Metabolic Syndrome.

311 HDL-C: High-Density Lipoprotein Cholesterol.

312 IMT: Intimal-Medial Thickness.

313 TIA: Transient Ischemic Attack.

314 CEA: Carotid Endarterectomy.

315 TCFA: Thin-Cap Fibro-Atheroma.

316 LDL-C: Low-Density Lipoprotein Cholesterol. 


\section{Declarations}

319 Ethics approval and consent to participate: Informed consent was obtained from each

320 patient. The study protocol was approved by the Institutional Review Board of the University

321 of Rome "Tor Vergata".

322

323 Consent for publication: All authors have agreed with the submission in its present (and

324 subsequent) forms.

325

326 Availability of data and materials: The data that support the findings of this study are

327 available from the corresponding author upon reasonable request.

328 Competing interests: The authors declare that they have no competing interests. The study is original, and the manuscript has not been published yet and is not being considered for 330 publication.

331 Funding: This study was not supported by specific funding.

332

333

Author Contributions: Conceptualization: F.S., L.A., M.C., M.F, and A.M.; Methodology: F.S., L.A., M.C., V.R., M.S., and M.M; Formal Analysis: F.S., M.M., E.G., R.B., and P.B.; Data Curation: M.C., M.S., M.M., I.M.L.and F.D.D.; Writing: Original Draft Preparation: F.S., L.A., and A.M.; Writing: Review and Editing: M.C., V.R., M.S., M.M., F.D.D., E.G., R.B., P.B., M.F., I.M.L., N.D.D. and A.M.; Supervision, M.F., N.D.D. and A.M. All authors have read and agreed to the published version of the manuscript.

Acknowledgements:

341 Not applicable. 


\section{References}

343 1. Alberti KG, Eckel RH, Grundy SM, Zimmet PZ, Cleeman JI, Donato KA, Fruchart JC, 344 James WP, Loria CM, Smith SC, Jr.: Harmonizing the metabolic syndrome: a joint interim statement of the International Diabetes Federation Task Force on Epidemiology and Prevention; National Heart, Lung, and Blood Institute; American Heart Association; World Heart Federation; International Atherosclerosis Society; and International Association for the Study of Obesity. Circulation 2009, 120(16):16401645.

2. Benjamin EJ, Muntner P, Alonso A, Bittencourt MS, Callaway CW, Carson AP, Chamberlain AM, Chang AR, Cheng S, Das SR et al: Heart Disease and Stroke Statistics-2019 Update: A Report From the American Heart Association. Circulation 2019, 139(10):e56-e528.

3. Li X, Fang F, Fu X, Lin H, Gao Q: Is Metabolic Syndrome Associated with the Risk of Recurrent Stroke: A Meta-Analysis of Cohort Studies. J Stroke Cerebrovasc Dis 2017, 26(12):2700-2705.

4. Roever L, Resende ES, Diniz ALD, Penha-Silva N, O'Connell JL, Gomes PFS, Zanetti HR, Roerver-Borges AS, Veloso FC, Fidale TM et al: Metabolic syndrome and risk of stroke: Protocol for an update systematic review and meta-analysis. Medicine (Baltimore) 2018, 97(15):e9862.

5. Gami AS, Witt BJ, Howard DE, Erwin PJ, Gami LA, Somers VK, Montori VM: Metabolic syndrome and risk of incident cardiovascular events and death: a systematic review and meta-analysis of longitudinal studies. J Am Coll Cardiol 2007, 49(4):403414.

6. Li X, Lin H, Fu X, Lin W, Li M, Zeng X, Gao Q: Metabolic syndrome and stroke: A meta-analysis of prospective cohort studies. J Clin Neurosci 2017, 40:34-38. 

JC, Horwitz RI: Impaired insulin sensitivity among nondiabetic patients with a recent TIA or ischemic stroke. Neurology 2003, 60(9):1447-1451.

8. Golden SH, Folsom AR, Coresh J, Sharrett AR, Szklo M, Brancati F: Risk factor groupings related to insulin resistance and their synergistic effects on subclinical atherosclerosis: the atherosclerosis risk in communities study. Diabetes 2002, 51(10):3069-3076.

374

9. Kernan WN, Viscoli CM, Furie KL, Young LH, Inzucchi SE, Gorman M, Guarino PD, Lovejoy AM, Peduzzi PN, Conwit R et al: Pioglitazone after Ischemic Stroke or Transient Ischemic Attack. N Engl J Med 2016, 374(14):1321-1331.

10. Cardellini M, Rizza S, Casagrande V, Cardolini I, Ballanti M, Davato F, Porzio O, 378 Canale MP, Legramante JM, Mavilio M et al: Soluble ST2 is a biomarker for cardiovascular mortality related to abnormal glucose metabolism in high-risk subjects. Acta Diabetol 2019, 56(3):273-280.

11. Hansson GK, Libby P: The immune response in atherosclerosis: a double-edged sword. Nat Rev Immunol 2006, 6(7):508-519.

12. Finn AV, Nakano M, Narula J, Kolodgie FD, Virmani R: Concept of vulnerable/unstable plaque. Arterioscler Thromb Vasc Biol 2010, 30(7):1282-1292.

13. Spagnoli LG, Mauriello A, Sangiorgi G, Fratoni S, Bonanno E, Schwartz RS, Piepgras DG, Pistolese R, Ippoliti A, Holmes DR, Jr.: Extracranial thrombotically active carotid plaque as a risk factor for ischemic stroke. JAMA 2004, 292(15):1845-1852.

14. Mauriello A, Servadei F, Sangiorgi G, Anemona L, Giacobbi E, Liotti D, Spagnoli LG: Asymptomatic carotid plaque rupture with unexpected thrombosis over a non-canonical vulnerable lesion. Atherosclerosis 2011, 218(2):356-362. 
391 15. Mauriello A, Sangiorgi GM, Virmani R, Trimarchi S, Holmes DR, Jr., Kolodgie FD, Piepgras DG, Piperno G, Liotti D, Narula J et al: A pathobiologic link between risk factors profile and morphological markers of carotid instability. Atherosclerosis 2010, 208(2):572-580.

16. Scimeca M, Anemona L, Granaglia A, Bonfiglio R, Urbano N, Toschi N, Santeusanio G, Schiaroli S, Mauriello S, Tancredi V et al: Plaque calcification is driven by different mechanisms of mineralization associated with specific cardiovascular risk factors. Nutr Metab Cardiovasc Dis 2019, 29(12):1330-1336.

17. Cardellini M, Rovella V, Scimeca M, Anemona L, Bischetti S, Casella S, Saggini A, Bonanno E, Ballanti M, Davato F et al: Chronic Kidney Disease Is Linked to Carotid Nodular Calcification, An Unstable Plaque Not Correlated to Inflammation. Aging Dis 2019, 10(1):71-81.

18. Kernan WN, Ovbiagele B, Black HR, Bravata DM, Chimowitz MI, Ezekowitz MD, Fang MC, Fisher M, Furie KL, Heck DV et al: Guidelines for the prevention of stroke in patients with stroke and transient ischemic attack: a guideline for healthcare professionals from the American Heart Association/American Stroke Association. Stroke 2014, 45(7):2160-2236.

19. Eckstein HH: European Society for Vascular Surgery Guidelines on the Management of Atherosclerotic Carotid and Vertebral Artery Disease. Eur J Vasc Endovasc Surg $2018,55(1): 1-2$.

20. Halliday A, Harrison M, Hayter E, Kong X, Mansfield A, Marro J, Pan H, Peto R, Potter J, Rahimi K et al: 10-year stroke prevention after successful carotid endarterectomy for asymptomatic stenosis (ACST-1): a multicentre randomised trial. Lancet 2010, 376(9746):1074-1084. 
415 21. Tsivgoulis G, Safouris A, Kim DE, Alexandrov AV: Recent Advances in Primary and Secondary Prevention of Atherosclerotic Stroke. J Stroke 2018, 20(2):145-166.

417

22. Guembe MJ, Fernandez-Lazaro CI, Sayon-Orea C, Toledo E, Moreno-Iribas C: Risk for cardiovascular disease associated with metabolic syndrome and its components: a 13-year prospective study in the RIVANA cohort. Cardiovasc Diabetol 2020, 19(1):195.

23. Kumar P, Sharma R, Misra S, Kumar A, Nath M, Nair P, Vibha D, Srivastava AK, Prasad K: CIMT as a risk factor for stroke subtype: A systematic review. Eur J Clin Invest 2020, 50(11):e13348.

24. Willeit P, Tschiderer L, Allara E, Reuber K, Seekircher L, Gao L, Liao X, Lonn E, Gerstein HC, Yusuf S et al: Carotid Intima-Media Thickness Progression as Surrogate

26. Whelton PK, Carey RM, Aronow WS, Casey DE, Jr., Collins KJ, Dennison

27. Williams B, Mancia G, Spiering W, Agabiti Rosei E, Azizi M, Burnier M, Clement DL, 
hypertension of the European Society of Cardiology and the European Society of Hypertension: The Task Force for the management of arterial hypertension of the European Society of Cardiology and the European Society of Hypertension. $J$ Hypertens 2018, 36(10):1953-2041.

28. Mauriello A, Sangiorgi G, Virmani R, Servadei F, Trimarchi S, Holmes DR, Jr., Kolodgie F, Biondi Zoccai G, Leuzzi C, Spagnoli LG: Evidence of a topographical link between unstable carotid plaques and luminal stenosis: can we better stratify asymptomatic patients with significant plaque burden? Int J Cardiol 2012, 155(2):309311.

29. Mottillo S, Filion KB, Genest J, Joseph L, Pilote L, Poirier P, Rinfret S, Schiffrin EL, Eisenberg MJ: The metabolic syndrome and cardiovascular risk a systematic review and meta-analysis. J Am Coll Cardiol 2010, 56(14):1113-1132.

30. Haffner SM, D'Agostino R, Jr., Mykkanen L, Tracy R, Howard B, Rewers M, Selby J, Savage PJ, Saad MF: Insulin sensitivity in subjects with type 2 diabetes. Relationship to cardiovascular risk factors: the Insulin Resistance Atherosclerosis Study. Diabetes Care 1999, 22(4):562-568.

31. Sheu WH, Jeng CY, Young MS, Le WJ, Chen YT: Coronary artery disease risk predicted by insulin resistance, plasma lipids, and hypertension in people without diabetes. Am J Med Sci 2000, 319(2):84-88.

32. Rovella V, Anemona L, Cardellini M, Scimeca M, Saggini A, Santeusanio G, Bonanno E, Montanaro M, Legramante IM, Ippoliti A et al: The role of obesity in carotid plaque instability: interaction with age, gender, and cardiovascular risk factors. Cardiovasc Diabetol 2018, 17(1):46.

33. Toth PP, Shah PK, Lepor NE: Targeting hypertriglyceridemia to mitigate cardiovascular risk: A review. Am J Prev Cardiol 2020, 3:100086. 
34. Schwartz GG, Abt M, Bao W, DeMicco D, Kallend D, Miller M, Mundl H, Olsson AG: Fasting triglycerides predict recurrent ischemic events in patients with acute coronary syndrome treated with statins. J Am Coll Cardiol 2015, 65(21):2267-2275.

35. Miller M, Cannon CP, Murphy SA, Qin J, Ray KK, Braunwald E: Impact of triglyceride levels beyond low-density lipoprotein cholesterol after acute coronary syndrome in the PROVE IT-TIMI 22 trial. J Am Coll Cardiol 2008, 51(7):724-730.

36. Bhatt DL, Steg PG, Miller M, Brinton EA, Jacobson TA, Ketchum SB, Doyle RT, Jr., Juliano RA, Jiao L, Granowitz C et al: Cardiovascular Risk Reduction with Icosapent Ethyl for Hypertriglyceridemia. N Engl J Med 2019, 380(1):11-22.

37. Marz W, Scharnagl H, Winkler K, Tiran A, Nauck M, Boehm BO, Winkelmann BR: Low-density lipoprotein triglycerides associated with low-grade systemic inflammation, adhesion molecules, and angiographic coronary artery disease: the Ludwigshafen Risk and Cardiovascular Health study. Circulation 2004, 110(19):30683074.

38. Nordestgaard BG: Triglyceride-Rich Lipoproteins and Atherosclerotic Cardiovascular Disease: New Insights From Epidemiology, Genetics, and Biology. Circ Res 2016, 118(4):547-563.

39. Doi H, Kugiyama K, Oka H, Sugiyama S, Ogata N, Koide SI, Nakamura SI, Yasue H: Remnant lipoproteins induce proatherothrombogenic molecules in endothelial cells through a redox-sensitive mechanism. Circulation 2000, 102(6):670-676.

40. Sangiorgi G, Roversi S, Biondi Zoccai G, Modena MG, Servadei F, Ippoliti A, Mauriello A: Sex-related differences in carotid plaque features and inflammation. $J$ Vasc Surg 2013, 57(2):338-344.

41. Burke AP, Farb A, Malcom G, Virmani R: Effect of menopause on plaque morphologic characteristics in coronary atherosclerosis. Am Heart J 2001, 141(2 Suppl):S58-62. 
490 42. Iemolo F, Martiniuk A, Steinman DA, Spence JD: Sex differences in carotid plaque 491 and stenosis. Stroke 2004, 35(2):477-481.

492 43. Manson JE, Hsia J, Johnson KC, Rossouw JE, Assaf AR, Lasser NL, Trevisan M, Black 493 HR, Heckbert SR, Detrano R et al: Estrogen plus progestin and the risk of coronary 494 heart disease. N Engl J Med 2003, 349(6):523-534.

495 44. Xing D, Nozell S, Chen YF, Hage F, Oparil S: Estrogen and mechanisms of vascular 496 protection. Arterioscler Thromb Vasc Biol 2009, 29(3):289-295.

497

498

499

500 
Gender

Male

Female

Cerebrovascular disease

Symptomatic patients

Ipsilateral major stroke

TIA

Asymptomatic patients

Risk factors

Hypertension AHA [26]

$162(87.1 \%)$

Hypertension ESC [27]

Diabetes

Smoking habit

Hypercholesterolemia

Hypertriglyceridemia
$131(70.4 \%)$

$55(29.6 \%)$

$74(39.8 \%)$

$44(23.7 \%)$

$30(16.1 \%)$

$112(60.2 \%)$
$80(43.0 \%)$

$40(21.5 \%)$

$29(15.6 \%)$

$70(37.6 \%)$ 
Low-HDL

$86(46.2 \%)$

IRC

$56(30.1 \%)$

Metabolic syndrome

$85(45.7 \%)$

Drugs

Statins

$128(68.8 \%)$

Diuretics

$153(82.3 \%)$

Associated vascular disease

Acute cardiovascular disease

$45(24.2 \%)$

Previous myocardial infarction

$37(19.9 \%)$

Instable angina

$8(4.3 \%)$

Peripheral arterial disease

$61(32.8 \%)$

Aortic aneurysm

$11(5.9 \%)$

Histological type of carotid plaque

Stable plaques

$104(55.9 \%)$

Fibroatheromata

$71(38.2 \%)$

Fibrocalcific

$33(17.7 \%)$

Unstable plaques

$82(44.1 \%)$

Thrombotic plaque

$48(25.8 \%)$

With a thrombus in organization

$16(8.6 \%)$

TCFA

$16(8.6 \%)$

Calcified nodule

$2(1.1 \%)$ 
502 Legend of Figures

503 Figure 1. Histology of carotid plaques

504 Panels A and B) Stable plaque characterized by a thick fibrous cap and a large lipidic necrotic

505 core with few inflammatory cells (Movat, 2x); Panels C and D) Unstable plaque constituted by

506 a thrombotic plaque associated to the cap rupture (Movat, 2x).

$507{ }^{*}$ Cap: fibrous cap; Ath: lipidic necrotic core; Thr: acute thrombus; Rup: site of cap rupture. 


\section{Figures}
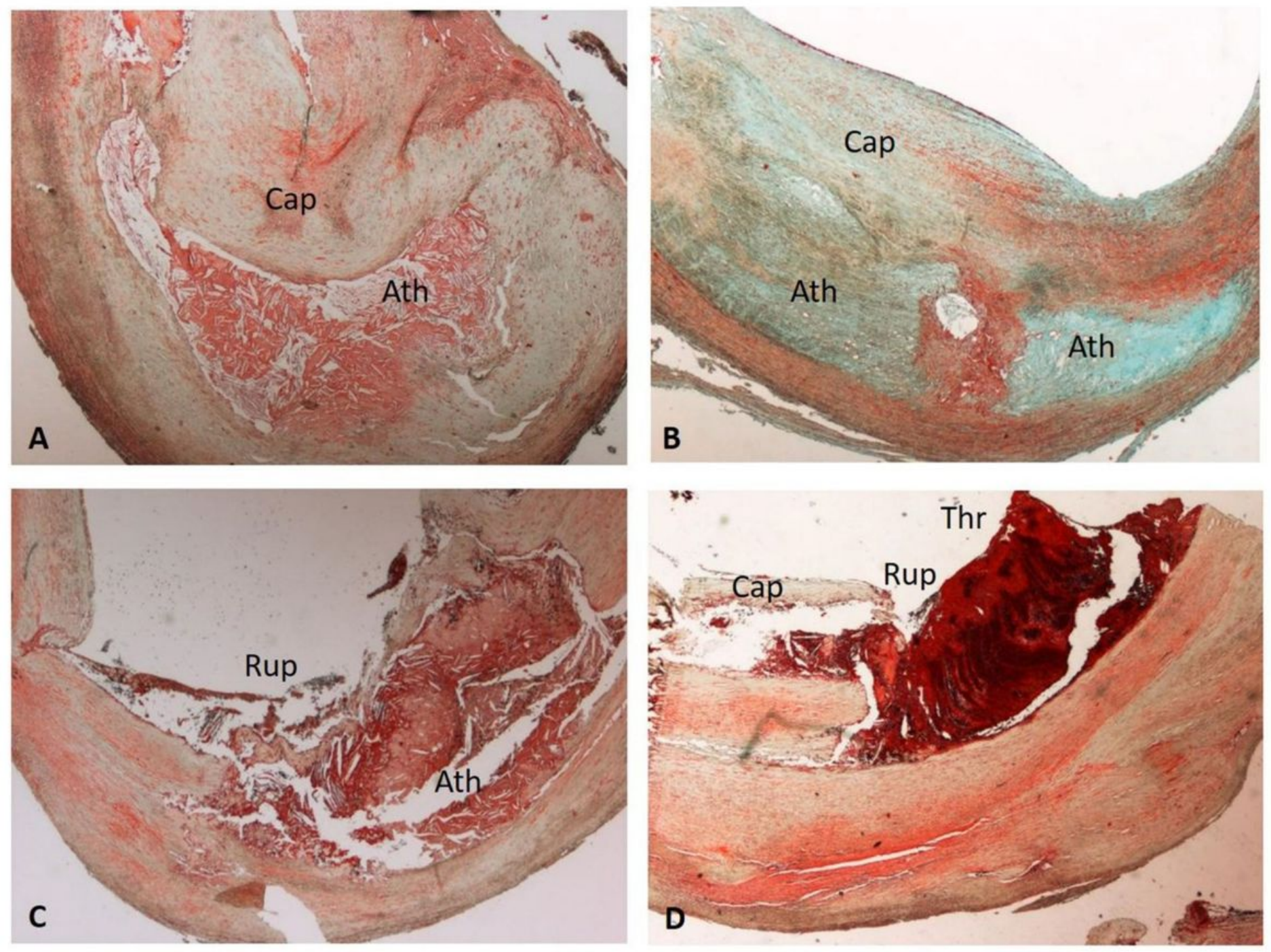

Figure 1

Histology of carotid plaques Panels A and B) Stable plaque characterized by a thick fibrous cap and a large lipidic necrotic core with few inflammatory cells (Movat, 2x); Panels $C$ and $D$ ) Unstable plaque constituted by a thrombotic plaque associated to the cap rupture (Movat, $2 x$ ). ${ }^{*}$ Cap: fibrous cap; Ath: lipidic necrotic core; Thr: acute thrombus; Rup: site of cap rupture. 